\title{
Effect of heat treatment condition on the phase formation of $\mathrm{YBa} 2 \mathrm{Cu} 3 \mathrm{O} 7-\delta$ superconductor
}

\begin{abstract}
Polycrystalline samples with the nominal composition $\mathrm{YBa} 2 \mathrm{Cu} 3 \mathrm{O} 7-\mathrm{u}(\mathrm{Y}-123)$ were prepared using the co-precipitation method. The effect of the calcination process (single and multiple calcinations) on the samples was investigated by using the four-point temperature-resistance measurement, x-ray diffraction (XRD) and field-emission scanning electron microscope (FESEM). This study is divided into two parts. For the first part, the obtained oxalate powder underwent two calcination processes at $900{ }^{\circ} \mathrm{C}$ for $12 \mathrm{~h}$ and $900{ }^{\circ} \mathrm{C}$ for $24 \mathrm{~h}$, respectively. Then, the powders were pressed into pellets and sintered at $920^{\circ} \mathrm{C}$ for $15 \mathrm{~h}$ with oxygen flow during the entire heat treatment. In the second part, only one calcination process was undertaken at $900{ }^{\circ} \mathrm{C}$ for $24 \mathrm{~h}$ before the sintering process in oxygen flow at $920{ }^{\circ} \mathrm{C}$ for $15 \mathrm{~h}$. From the XRD patterns, all of the peaks were indexed to the Y-123 phase showing that this superconducting phase was already formed after the first calcination. The volume fraction of Y-123 of the samples with single calcination process was higher compared to multiple calcination processes. From the temperature-resistance measurement, all the samples showed metallic behavior in the normal state and a superconducting transition to zero resistance. The superconducting transition temperature, Tc, for the samples prepared in a single calcination process is higher than that of the multiple calcination processes.
\end{abstract}

Keyword: Co-precipitation; FESEM; Superconductivity; XRD; YBa2Cu3O7-ŭ 